Internist 2017 $\cdot 58: 643$

DOI 10.1007/s00108-017-0233-0

Online publiziert: 10. Mai 2017

(c) Springer Medizin Verlag GmbH 2017

CrossMark

\title{
G. Schultze
}

Villingen-Schwenningen, Deutschland

\section{"Chronic kidney disease" ungleich chronische Niereninsuffizienz}

te bestehen und Implikationen für die Gesundheit haben.

Kriterien für eine chronische Nierenkrankheit sind Marker für einen Nierenschaden wie Albuminurie, anomales Urinsediment, Elektrolytanomalien durch tubuläre Schäden, anomale Histologie, Anomalien in der Bildgebung und $\mathrm{Zu}$ stand nach Nierentransplantation sowie eine reduzierte $G F R<60 \mathrm{ml} / \mathrm{min} / 1,73 \mathrm{~m}^{2}$ (GFR-Kategorien G3a-G5).

Die Klassifikation der chronischen Nierenkrankheit sollte auf Basis der Ursache, der GFR-Kategorie und der Kategorie der Albuminurie erfolgen.

Die Ursache der chronischen Nierenkrankheit sollte auf dem Vorliegen oder Nichtvorliegen einer Systemerkrankung und der Lokalisierung von beobachteten oder angenommen pathologisch-anatomischen Befunden innerhalb der Nieren basieren.

Die GFR-Kategorien ( $\left.\mathrm{ml} / \mathrm{min} / 1,73 \mathrm{~m}^{2}\right)$ sind folgende:

- G1: $\geq 90$; normal oder hoch,

- G2: 60-89; leicht vermindert,

- G3a: 45-59; leicht bis mäßig vermindert,

- G3b: 30-44; mäßig bis schwer vermindert,

- G4: 15-29; schwer vermindert,

- G5: Nierenversagen.

In einer Fußnote wird ausdrücklich darauf hingewiesen, dass die Kategorien G1 und G2 allein nicht die Kriterien für eine chronische Nierenerkrankung erfüllen. Ein Patient mit der Kategorie G3 hat also nicht das Stadium 3 einer Niereninsuffizienz, sondern eine chronische Nierenerkrankung mit der GFR-Kategorie G3. Das mag spitzfindig klingen. Stadium 3 einer
Niereninsuffizienz suggeriert aber, dass bei 5 Stadien das dritte Stadium schon eine weit fortgeschrittene Insuffizienz bedeutet. Dabei zeigt G3a erst eine milde bis moderate Verminderung an.

Außerdem kann ein Patient auch bei normaler glomerulärer Filtrationsrate eine Nierenkrankheit haben.

Mit freundlichen Grüßen

G. Schultze

\section{Korrespondenzadresse}

Prof. Dr. G. Schultze

Hubertusweg 8, 78048 Villingen-Schwenningen, Deutschland

gschultze.vs@t-online.de

Interessenkonflikt. G. Schultze gibt an, dass kein Interessenkonflikt besteht.
„Die chronische Nierenkrankheit („,chronic kidney disease" [CKD]) ist definiert als Abnormitäten von Strukturen und Funktionen der Nieren, die länger als 3 Mona- 